\title{
Effects of inter-twin vascular anastomoses of monochorionic twins with selective intrauterine growth restriction on the contents of placental mitochondria DNA
}

Yao-Lung Chang ${ }^{1}$, An-Shine Chao ${ }^{1}$, Hsiu-Huei Peng ${ }^{1}$, Shuenn-Dyh Chang ${ }^{1}$, Sheng-Yuan Su', Kuan-Ju Chen ${ }^{1}$ and Tzu-Hao Wang ${ }^{1,2,3^{*}}$

\begin{abstract}
Background: Placental mitochondrial DNA (mtDNA) has been proposed to be an indicator for placental hypoxia. This study was designed to evaluate the effect of vascular anastomoses between monochorionic (MC) twins on placental mtDNA.

Methods: In this study, twin-twin transfusion syndrome (TTS) treated with laser therapy and MC twins without TTS (without laser therapy) resulting in two live babies were included in this study. The placental mtDNA fold changes (FC) between the small and large twins were analyzed using real-time quantitative PCR. TTS twins with selective intrauterine growth restriction (SIUGR) are categorized as group 1, TाTS without sIUGR as group 2, MC twins without TTS but with sIUGR as group 3, and MC twins without both TITS and sIUGR as group 4.

Results: There were seven cases in group 1, eight in group 2, 26 in group 3, and 24 in group 4 cases. The placental mtDNA FC were significantly higher in group 1 (1.57 \pm 0.9$)$ compared to that of the group $3(0.86 \pm 0.6)$.

Conclusion: In MC twin pregnancies with sIUGR, the placental mtDNA FC between the small and large twins are different between cases with and without inter-twin anastomoses. These findings suggest that the inter-twin anastomoses in the MC twins with sIUGR may provide rescue perfusion from the appropriate-for-gestational-age twin to the sIUGR one.
\end{abstract}

Keywords: Placenta mitochondria, Monochorionic twins, Twin-twin transfusion syndrome

\section{Background}

Every mitochondrion contains $2-10$ copies of mitochondrial DNA (mtDNA) in its matrix, resulting in more than 10,000 copies of mtDNA in each human cell [1-3]. MtDNA typically replicates during every cell cycle; each daughter cell usually maintains a relatively consistent number of copies of mtDNA [4]. However, replication of mitochondrial DNA may also be independently affected

\footnotetext{
* Correspondence: knoxtn@cgmh.org.tw

'Department of Obstetrics and Gynecology, Chang Gung Memorial Hospital, Linkou, College of Medicine, Chang Gung University, No 5, Fu-Shin Road, Gwei-shan, Taoyuan, Taiwan

${ }^{2}$ School of Traditional Chinese Medicine, College of Medicine, Chang Gung University, Taoyuan, Taiwan

Full list of author information is available at the end of the article
}

by genetic disorders [5] or environmental factors, such as oxidative stress $[6,7]$.

MtDNA has been shown to be increased in placental tissues of pregnancies with intrauterine growth restriction (IUGR) $[8,9]$. Although the exact mechanism how the placental mtDNA increase in the case of sIUGR remains unclear, placental mtDNA contents were correlated with low oxygen levels [8]. Furthermore, the copy numbers of mitochondrial DNA were increased by changes affecting fetal growth [10]. Monochorionic (MC) twins, who share identical genetic materials, enable us to study the mechanisms of mtDNA increase in cases of IUGR without confounding effects from genetic factors.

There are three types of intertwin anastomoses in monochorionic twins: artery-to-artery (AA), vein-to-vein 
(VV), and artery-to-vein (AV) anastomoses. AA and VV anastomoses form direct communications on the surface of the chorionic plate and are bidirectional. Unidirectional artery-to-vein (AV) anastomoses are located deep in the placental tissue. An imbalance in intertwin transfusion through vascular anastomoses is the underlying mechanism of TTTS [11]. An effective treatment of twin-twin transfusion syndrome (TTTS) is to disrupt the vascular anastomoses in the placenta of $\mathrm{MC}$ twins with laser photocoagulation therapy $[12,13]$. However, about $41.4 \%$ of TTTS suffer from selective FGR (sIUGR) even after successful laser therapy resulting in two live twins [14].

The development of sIUGR in MC twins is likely due to unequal placental sharing [15]. We have previously found that mitochondrial activation in cord blood and the amniocytes of the sIUGR fetus in MC twins was likely to be regulated by locally adverse placental conditions resulting in reduced blood flow, rather than genetic factors [16].

In the current study to further test the hypothesis that the twin-twin vascular anastomoses may provide a rescue perfusion to the sIUGR twin, we attempted to compare the mtDNA quantity in the placental territories of each fetus in MC twin pregnancies with or without sIUGR. Furthermore, we studied the changes in placental mtDNA contents after laser therapy for the treatment of TTTS.

\section{Methods}

\section{Clinical subjects}

In this study, we only analyzed the women whom ultimately gave birth at Chang Gung Memorial Hospital, Taoyuan, Taiwan, which is a tertiary referral center with 3668 beds. All women included into this study were Taiwanese. All of the MC twin gestations with TTTS during the period of this study were treated with laser therapy, and all of these pregnancies were delivered by Cesarean section.

The inclusion criteria of this study included: TTTS twins successfully treated by laser therapy resulting in two live infants with placentas that were intact enough to be studied after birth. Other MC twins without TTTS, with or without sIUGR, were also enrolled in the control group. Successful laser therapy for TTTS was defined as the resolution of polyhydramnios-oligohydramnios after the procedure, delivery of two viable babies, and no obvious residual superficial anastomoses detected via a thorough gross placental examination at delivery. Monoamniotic twin are excluded from this study.

A twin pregnancy with sIUGR was defined as an estimated fetal weight below the 10th percentile for gestational age in one twin according to a singleton growth chart, whereas the appropriate-for-gestational-age (AGA) twin was the co-twin without FGR [17]. The diagnosis of TTTS was based on the ultrasound findings defined by Quintero et al. [12]. The laser therapy were performed between TTTS diagnosed at gestational 16 26 weeks. In
TTTS twins or MC twins without TTTS and without sIUGR (group 4), the larger twin is the one with heavier birth weight. The discordance between the twins' weights was defined as (birth weight of the larger twin - birth weight of the smaller twin) $\times 100 \% /$ (birth weight of the larger twin). The discordance of placental shares was calculated similarly (see below for further details).

\section{Inspection and collection of placental tissues}

The placentas were collected immediately after delivery. After blood was drained from the umbilical vessels, the placenta was washed with ice-cold PBS to remove all blood clots and examined for integrity of the cotyledons and membranes. The placenta was weighed after the umbilical cord and membranes were removed. In cases without TTTS, the vascular equator was defined as a border drawn in the middle of the vascular zone on the chorionic fetal surface where the communicating vessels met. In cases with TTTS, the vascular equator was defined as the area where laser therapy had been applied to coagulate anastomoses. If residual anastomosis was detected by visual inspection, the placenta was excluded from this study. The placentas were cut along the vascular equator. Each placental portion was weighed separately to record the individual placenta mass (IPM) [18]. The discordance of placental shares was calculated as (IPM of the larger twin - IPM of the smaller twin) $\times 100 \% /$ (IPM of the larger twin).

We collected two to three biopsies of fresh placental tissue $(0.5 \mathrm{X} 0.5 \mathrm{X} 0.5 \mathrm{~cm})$ from each placental territory of each MC twin. The placental tissue biopsies were taken at the midway point between the vascular equator to its corresponding cord insertion, and at the middle layer of placenta between the maternal and fetal surfaces. Regions with obvious calcification or infarction were avoided. The placenta specimens were briefly rinsed with ice-cold PBS to remove blood, snap frozen in liquid nitrogen, and stored in a $-70{ }^{\circ} \mathrm{C}$ freezer.

Real-time quantitative polymerase chain reaction (PCR) analysis.

The protocols of mtDNA real time quantitative PCR using TaqMan PCR Core Reagent Kit and the Sequence Detection system (ABI Prism 7900, Applied Biosystems) have been previously reported [5]. Briefly, the ABI mitochondrial gene 7S encoding D-loop was used as the target gene (Hs02596861_s1, Applied Biosystems, CA, USA), and the amount of glyceraldehyde-3-phosphate dehydrogenese (GAPDH) gene was used to represent nuclear DNA concentrations. Thermal cycling was initiated with 2-min incubation at $50{ }^{\circ} \mathrm{C}$ and followed by the first denaturation period of $10 \mathrm{~min}$ at $95^{\circ} \mathrm{C}$, followed by 40 cycles of $95^{\circ} \mathrm{C}$ for $15 \mathrm{~s}$ and $60{ }^{\circ} \mathrm{C}$ for 1 minute. In our laboratory, we routinely do RT-QPCR of each sample in duplicate and keep the coefficient of variation (CV) less than $15 \%$. Whenever the CV of two values appears greater than 15\% (it hardly 
happens), we discard that measurement and re-assay the sample. The comparative threshold cycle $(\mathrm{Ct})$ method was used to analyze data [19]. The $\Delta \mathrm{Ct}$ values from each sample were obtained by subtracting the value of endogenous control gene. The $2^{-\Delta \Delta \mathrm{Ct}}$ was used to calculate relative amounts of mtDNA, represented as the placental mtDNA fold changes, between the two fetuses in MC twins.

\section{Data analysis}

The placental mitochondrial DNA (mtDNA) fold change was defined as (placental mtDNA content of the sIUGR or smaller fetus) / (placental mtDNA content of the AGA or larger fetus). The placental mtDNA fold change in each pair of MC twins was calculated, then those data were analyzed with the SPSS software, generating means and standard deviation (S.D.) in each one of the four groups of subjects.

Statistical analysis was conducted with the SPSS software (version 11.0 for Window; SPSS Inc, Chicago, IL). Continuous variables were first tested for normal distribution using the Kolmogorov-Smirnov test. Two-sample Student $t$ test or Mann-Whitney $U$ test were used to compare the values of two groups, binomial factor were compared by Chi-Square test and one-way ANOVA test or Kruskal-Wallis Test (if the $p$ value of Test of Homogeneity of Variances was $<0.05$ ) were used for data more than or equal to three groups. A probability value of less than 0.05 was considered to be statistically significant.

\section{Results}

From July 2010 to June 2014, 65 pairs of MC twins were included in this study, including 15 pairs with TTTS and 50 without TTTS (named as No TTTS). The mean gestational age of laser therapy for TTTS was $20 \pm 2.7$ weeks of gestation. In the group of TTTS (group 1 and 2); there were two cases as Quintero stage I, six as stage II, six as stage III, and one as stage IV, resulting in 7 pairs with sIUGR (group 1) and 8 pairs without (group 2). In the group of MC twins without TTTS, 26 cases were found to have sIUGR (group 3) and 24 cases did not develop sIUGR (group 4). The percentage of sIUGR in MC twins at our hospital, a tertiary referral center, was $52 \%$ (26/50). There were no statistical differences in maternal age at delivery, gestational age at delivery, birth weight of the smaller twin, birth weight of the larger twin, or birth weight discordance between TTTS and No TTTS twins, in cases with sIUGR (group 1 vs. group 3, Table 1 , $P 1$ ) or in the cases without sIUGR (group 2 vs. group 4, Table 1,P2). Because pregnancies with sIUGR were generally delivered earlier, the fetal birth weight of groups 1 and 3 were lighter than that of groups 2 and 4 (groups $1+3$ vs. groups $2+4$ Table $1, P 3$ ). Birth weight discordance was significantly higher in twins with sIUGR than those without (Table 1, P3).
All of MC twins without TTTS (group 3 and 4) were found have patent inter-twin anastomoses in placental inspection. There was no residual inter-twin anastomosis found by inspection in cases of TTTS post laser therapy. There was a significant difference in placental mtDNA fold changes (FC) among the four groups of twins (one-way ANOVA test, $p=0.035$ ). Post- hoc comparison revealed that the placental mtDNA FC was significantly higher in TTTS with sIUGR (group 1) than in the no TTTS twins with sIUGR (group 3) (least significant difference, LSD test, $p=0.008)$, but the difference was not significant between group 2 and group 4 (Fig. 1). Interestingly, the no TTTS twins with sIUGR (group 3 ) exhibited a significantly lower number of placental mtDNA FC than the no TTTS twins without sIUGR (group 4) (LSD, $p=0.039$ ). Of note, although the averaged FC in group 3 was less than $1(0.86)$, the difference between placental mtDNA amount in the sIUGR and that in the AGA twins was not statistically significant $(p=0.228)$.

To exclude the possibility that the difference in placental mtDNA FC between group 1 and group 3 twins was caused by discordance in the different placental shares, we also compared the placenta share discordance between groups 1 and 3 twins, and between groups 2 and 4 (Fig. 2). There were no significant differences in the placental weight discordance between groups 1 and 3 twin pregnancies which were the groups with sIUGR $(P=0.617)$ or between groups 2 and 4 twin pregnancies which were the groups without sIUGR $(P=0.741)$.

\section{Discussion}

The fetoscopic laser therapy [20] is currently recognized as the first-line treatment for stage I to IV TTTS diagnosed before 26 weeks of gestation, resulting in better survival and neurological outcomes of fetuses. However, because laser therapy has to coagulate the placental anastomotic vessels between the two fetuses in TTTS, the decreased placental perfusion to the donor twin with IUGR may cause a higher fetal demise rate than in the TTTS donor without IUGR [21]. Furthermore, the procedure of laser therapy is not free of complications. In our series, $11.4 \%$ of treated cases experienced premature rupture of membranes (PROMs) within 3 weeks, and 2.2\% of treated cases developed chorioamnionitis needing termination [13]. So in the TTTS twins where the donor has IUGR and abnormal UA Doppler, the poorer fetal outcomes should be discussed with the pregnant woman. In laser therapy for TTTS with sIUGR, it is important to preserve the blood vessels supplying to the donor twin as much as possible. In the sequential selective laser photocoagulation of communicating vessels (SQLPCV) technique, occlusion of the vascular communications is done in a particular order, resulting in a transient intra-operative net transfusion to the donor twin and facilitating a better hemodynamic equilibrium [22]. SQLPCV 
Table 1 Characteristics of the four groups of twin pregnancies

\begin{tabular}{|c|c|c|c|c|c|c|c|c|}
\hline & $\begin{array}{c}\text { Group 1 } \\
(\mathrm{n}=7)\end{array}$ & $\begin{array}{c}\text { Group } 2 \\
(\mathrm{n}=8)\end{array}$ & $\begin{array}{c}\text { Group } 3 \\
(\mathrm{n}=26)\end{array}$ & $\begin{array}{c}\text { Group } 4 \\
(\mathrm{n}=24)\end{array}$ & $p$ & $P 1$ & $P 2$ & $P 3$ \\
\hline $\begin{array}{l}\text { Maternal age } \\
\text { (years) }\end{array}$ & $\begin{array}{r}32.8 \\
\pm 4.9\end{array}$ & $\begin{array}{l}33.2 \\
\pm 2.5\end{array}$ & $\begin{array}{r}30.3 \\
\pm 2.1\end{array}$ & $\begin{array}{l}31.4 \\
\pm 4.4\end{array}$ & $0.078^{\&}$ & $0.12^{\%}$ & $0.215^{\#}$ & $0.48^{\#}$ \\
\hline $\begin{array}{l}\text { GA at } \\
\text { delivery } \\
\text { (weeks) }\end{array}$ & $\begin{array}{r}33.4 \\
\pm 3.2\end{array}$ & $\begin{array}{r}35.3 \\
\pm 0.9\end{array}$ & $\begin{array}{l}33.4 \\
\pm 2.7\end{array}$ & $\begin{array}{l}35.8 \\
\pm 2.1\end{array}$ & $0.124^{*}$ & $0.75^{\%}$ & $0.086^{*}$ & $<0.001$ \# \\
\hline $\begin{array}{l}\text { BW of AGA } \\
\text { twin (g) }\end{array}$ & $\begin{array}{l}1908 \\
\pm 406\end{array}$ & $\begin{array}{r}2269 \\
\pm 211\end{array}$ & $\begin{array}{r}1975 \\
\pm 528\end{array}$ & $\begin{array}{l}2283 \\
\pm 344\end{array}$ & $0.058^{\&}$ & $0.48^{*}$ & $0.623 \#$ & $0.004 \#$ \\
\hline $\begin{array}{l}\text { BW of } \\
\text { sIUGR twin } \\
\text { (g) }\end{array}$ & $\begin{array}{c}1179 \pm \\
268\end{array}$ & $\begin{array}{r}2135 \\
\pm 257\end{array}$ & $\begin{array}{l}1320 \\
\pm 498\end{array}$ & $\begin{array}{l}2157 \\
\pm 326\end{array}$ & $<0.001^{\&}$ & $0.78^{*}$ & $0.78^{*}$ & $<0.001 \#$ \\
\hline $\begin{array}{l}\text { BW } \\
\text { discordance } \\
(\%)\end{array}$ & $\begin{array}{r}37.7 \\
\pm 9.8\end{array}$ & $\begin{array}{c}6.1 \\
\pm 4.6\end{array}$ & $\begin{array}{c}34.7 \\
\pm 11.8\end{array}$ & $\begin{array}{c}5.5 \\
\pm 4.3\end{array}$ & $<0.001^{\&}$ & $0.54 \#$ & $0.633^{\#}$ & $<0.001$ \# \\
\hline $\begin{array}{l}\text { Preeclampsia } \\
\text { (N) }\end{array}$ & 0 & 0 & 2 & 3 & $0.238^{\S}$ & & & \\
\hline GDM (N) & 0 & 0 & 3 & 4 & $0.238^{8}$ & & & \\
\hline
\end{tabular}

Group 1: TTTS with sIUGR, group 2: TTS without sIUGR, group 3: No TTS twin with sIUGR, group 4: No TTS twin without sIUGR.

$p: p$ value among the four groups of twin pregnancies generate by one-way ANOVA test or Kruskal-Wallis Test

$P 1: P$ value between TTTS with sIUGR (group 1 ) and No TTS with sIUGR (group 3)

$P 2: P$ value between TTTS without sIUGR (group 2) and No TTS without sIUGR (group 4)

P3: $P$ value between twin pregnancies with sIUGR (groups $1+3$ ) and without sIUGR (groups $2+4$ )

Data are presented as Mean \pm SD except the number of preeclampsia and gestational diabetes mellitus

*one-way ANOVA test,

${ }^{\&}$ Kruskal-Wallis Test

*Mann-Whitney U test,

"Student $t$ test

${ }^{5}$ Chi-square test

may be the laser strategy of choice for managing the intertwin anastomoses for TTTS with sIUGR.

After laser coagulation of the inter-twin anastomoses in the placenta of TTTS twins, the placenta became functionally dichorionic, resulting in the resolution of the polyhydramnios-oligohydramnios sequence. Group 1 twins are very similar to monozygotic, dichorionic twins with sIUGR, in which the two fetuses share identical DNA content without twin-twin vascular anastomoses since they have two separate placentas, but one fetus develops IUGR. In group 1 twins, placental mtDNA contents were 1.57-fold higher in the placental territory of the sIUGR twin than that of the larger twin (Fig. 1). Our findings were similar to previous findings in singleton pregnancies, in which placental mtDNA contents are higher in pregnancies with IUGR than in normal pregnancies [8].

Our findings that placental mtDNA fold changes were significantly higher in group 1 (those without patent vascular anastomoses) than in the group 3 twin pregnancies (those with patent vascular anastomoses) indicate that blood shunting may temper the increase of the placental mtDNA content in the sIUGR twin. MtDNA increases have been associated with hypoxia in the placentas of IUGR [8]. Our results further suggest that the rescue perfusion from the AGA- to sIUGR-fetuses determines the placental hypoxic status in the sIUGR twins between these two groups. 


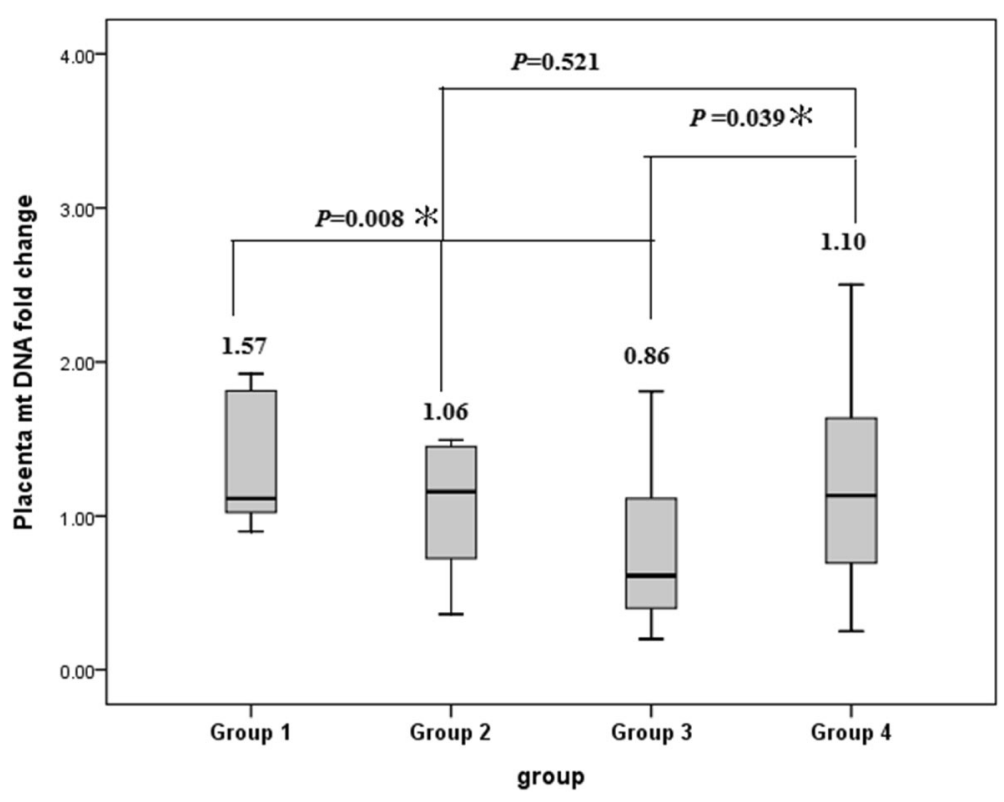

Fig. 1 Placental mtDNA fold changes in 4 groups of monochorionic twin pregnancies. The placental mitochondrial DNA (mtDNA) fold change was defined as (placental mtDNA content of the sIUGR or smaller fetus) / (placental mtDNA content of the AGA or larger fetus). Statistics are graphically presented as the median (horizontal bar), 25 and 75 percentile (box), and \pm 2 standard deviations (whiskers). Means of each group of data are also indicated in numeric figures. Grouping: Group 1: TTTS with sIUGR, Group 2: TTTS without sIUGR, Group 3: No TTTS with sIUGR, Group 4: No TTTS without sIUGR. Abbreviations: TTTS: twin-twin transfusion syndrome, sIUGR: selective intrauterine growth restriction, MC: monochorionic. *: $P$ value $<0.05$

One potential etiology of sIUGR in MC twins is its low placental share [14]. Our results (Fig. 2) of similar placental discordance in the TTTS with sIUGR (group 1) and the no TTTS twins with sIUGR (group 3) provided evidence against the hypothesis that the different degrees of low placental share in the sIUGR twins led to different placental mtDNA contents between these two groups.

We have previously reported that the fetal / placenta weight $(\mathrm{F} / \mathrm{P})$ ratio is increased in MC twins with sIUGR [23] when rescue perfusion from the larger placental territory to

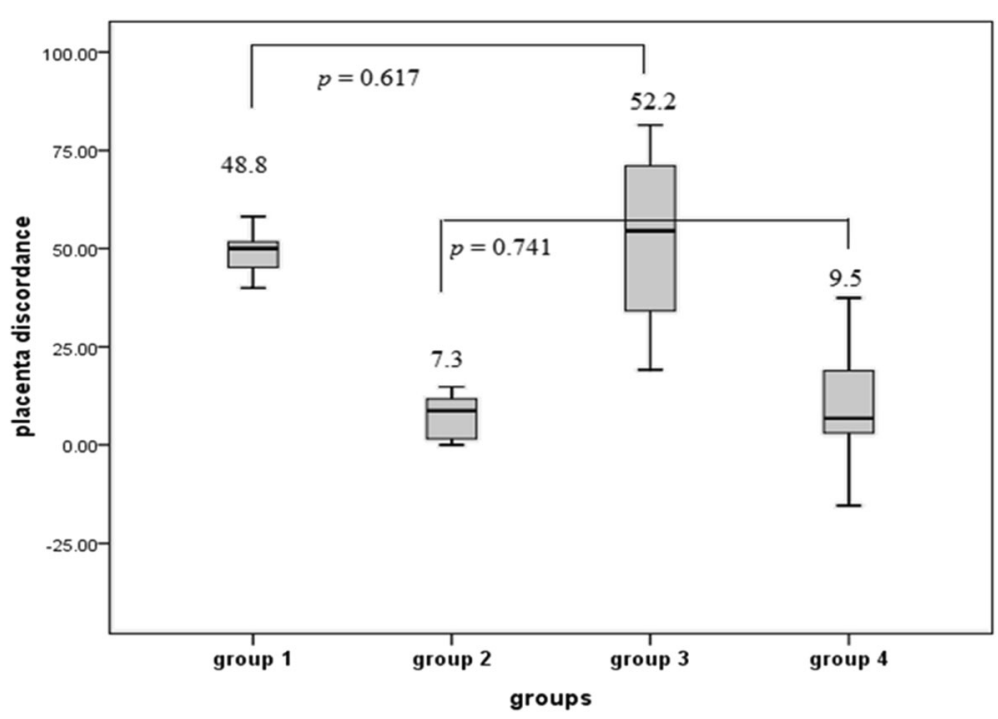

Fig. 2 The placental discordance of four groups of monochorionic twin pregnancies. The discordance of placental shares between twins was defined as (individual placenta mass of the larger twin -individual placenta mass of the smaller twin) $\times 100 \%$ / (individual placenta mass of the larger twin). Statistics are graphically presented as the median (horizontal bar), 25 and 75 percentile (box), and \pm 2 standard deviations (whiskers). Means of each group of data are also indicated in numeric figures. Grouping: Group 1: TTTS with sIUGR, Group 2: TTTS without sIUGR, Group 3: No TTS with sIUGR, Group 4: No TTS without SIUGR. Abbreviations: TTS: twin-twin transfusion syndrome, sIUGR: selective intrauterine growth restriction, MC: monochorionic. *: $P$ value $<0.05$ 
the sIUGR one through vascular anastomoses works to promote growth of the sIUGR twin. By this compensatory mechanism, the sIUGR twin in each pair of shunt-patent twins (group 3) gets more perfusion than its small placental territory would usually supply, resulting in the alleviation of hypoxic stressors and a decrease of mtDNA content in the sIUGR placental territory. On the other hand, laser therapy disrupts the vascular shunt between the TTTS twins and terminates the aforementioned rescue perfusion, resulting in hypoxia and, thus, increased mtDNA contents in the placental territory of the sIUGR twin. The placental mtDNA fold change between the smaller and larger twins was not different between group 2 and 4 twin pregnancies (Fig. 1). This finding shows that, in MC twins without sIUGR but with TTTS (group 2), blocking the vascular anastomoses to treat TTTS did not cause hypoxia in the placental territory of either twin and did not increase the placental mtDNA content.

It is important to point out that the fetal blood of the sIUGR twin in MC twins contains significantly higher mtDNA [16], representing a higher state of stress in the sIUGR fetus. However, the hypoxic status of the placental compartment is quite different. In the presence of vascular anastomoses between the MC twins without TTTS (which do not require laser therapy), the presence of sIUGR (group 3) triggers a rescue shunt that effectively relieves hypoxia resulting in decreased placental mtDNA fold changes.

Limitations of this study do exist. First, the case number in the TTTS with sIUGR in this study is small $(n=7)$. After laser therapy, the survival rate of both twins in MC pregnancies complicated by TTTS in our center was around $55 \%(55 / 100)$, and the survival rate was further decreased to $33.3 \%(12 / 36)$ when the TTTS twins were complicated by sIUGR in our center [21,24]. Therefore, it was not easy to collect the 7 cases of TTTS with sIUGR with both twins surviving after laser therapy and with intact placentas after delivery. Second, we could not exclude the possibility that the increased placental mtDNA fold changes in group 1 had been caused by the insult of polyhydramniosoligohydramnios before laser therapy, although the demographic data between groups 1 and 3 were similar (Table 1, P1). The hypothetical detrimental effects of polyhydramnios-oligohydramnios was further weakened by the findings of similar placental mtDNA fold changes between groups 2 and 4 (Fig. 1). Third, we carefully examined every placenta with visual inspection, but did not perform dye-injection study. Therefore, residual anastomoses, especially tiny ones, cannot be completely ruled out. Lastly, rinsing the placental tissues with ice-cold PBS might not completely remove the contamination of maternal blood and thereby maternal mtDNA. However, since the comparison was done in each pair of MC twins, the effect of contamination by maternal mtDNA was equally on each twin, thus being minimized. The consideration again supports the advantage of using MC twins for sIUGR studies.

\section{Conclusion}

In MC twin with sIUGR, the placental mtDNA fold changes between the small and large twins are different between cases with and without inter-twin anastomoses. We suspect that upon the development of sIUGR in MC twins, twin-twin vascular anastomoses in the placenta serve as a mechanism for rescue perfusion from the placental territory of the AGA twin to that of sIUGR. This compensatory mechanism alleviates the placental hypoxia in the placental territory of sIUGR twin.

\section{Additional file}

Additional file 1: Placenta mitochondria data. Code: experiment code of patients. Quintero stage: Quintero stage of TTS cases. Group: groups of cases (Group 1: TTS with sIUGR, Group 2: TाTS without sIUGR, Group 3: No TITS with sIUGR, Group 4: No TITS without sIUGR. GAD: gestational age at delivery. Birth weight (small): birth weight of smaller or sIUGR fetus. Birth weight (large): birth weight of larger of AGA fetus. Fold change: The placenta mtDNA fold change: was defined as (placental mtDNA content of the sIUGR or smaller fetus) / (placental mtDNA content of the AGA or larger fetus). (XLSX $16 \mathrm{~kb}$ )

\section{Abbreviations}

AGA: Appropriate-for-gestational-age; F/P ratio: Fetal/placenta ratio; FC: Fold changes; MC: Monochorionic; mtDNA: Mitochondrial DNA; sIUGR: Selective intrauterine growth restriction; TTS: Twin-twin transfusion syndrome

\section{Acknowledgments}

The authors thank technical assistance of Hsuen-Yu Kuo and Pei-Chi Wei (Chang Gung Memorial Hospital).

\section{Funding}

This work was supported by CMRPG3D0181 from Chang Gung Memorial Hospital, Linkou Medical Center. The role of the CMRPG3D0181 was to support the collections of specimens and laboratory procedures.

\section{Availability of data and materials}

The data that support the findings of this study including the maternal age at delivery, Quintero stage of TTS cases, groups of patients, birth weight of fetuses and placenta mtDNA fold changes are available in the Additional file 1.

\section{Authors' contributions}

All authors have contributed to study design and drafting or revising the manuscript, approved this version to be published, and are willing to take public responsibility for the accuracy and integrity of its content.

\section{Ethics approval and consent to participate}

With an approval by the Institute Review Board of Chang Gung Memorial Hospital (IRB \# 98-0669B and \#102-2979A3), this study was prospectively carried out in patients with MC twin gestations. Written informed consents have been obtained from all participants, and no participant was under the age of 16.

\section{Consent for publication}

Not applicable.

\section{Competing interests}

All of the authors declare that they have no competing interests.

\section{Publisher's Note}

Springer Nature remains neutral with regard to jurisdictional claims in published maps and institutional affiliations. 


\section{Author details}

'Department of Obstetrics and Gynecology, Chang Gung Memorial Hospital, Linkou, College of Medicine, Chang Gung University, No 5, Fu-Shin Road, Gwei-shan, Taoyuan, Taiwan. ${ }^{2}$ School of Traditional Chinese Medicine, College of Medicine, Chang Gung University, Taoyuan, Taiwan. ${ }^{3}$ Genomic Medicine Research Core Laboratory (GMRCL), Chang Gung Memorial Hospital, Tao-Yuan 333, Taiwan.

\section{Received: 10 October 2017 Accepted: 15 March 2018}

Published online: 24 March 2018

\section{References}

1. Kaaman M, Sparks LM, van Harmelen V, Smith SR, Sjolin E, Dahlman I, Arner P. Strong association between mitochondrial DNA copy number and lipogenesis in human white adipose tissue. Diabetologia. 2007;50(12):2526-33.

2. Petit C, Mathez D, Barthelemy C, Leste-Lasserre T, Naviaux RK, Sonigo P, Leibowitch J. Quantitation of blood lymphocyte mitochondrial DNA for the monitoring of antiretroviral drug-induced mitochondrial DNA depletion. J Acquir Immune Defic Syndr. 2003;33(4):461-9.

3. Wiesner RJ, Ruegg JC, Morano I. Counting target molecules by exponential polymerase chain reaction: copy number of mitochondrial DNA in rat tissues. Biochem Biophys Res Commun. 1992;183(2):553-9.

4. Lee HC, Wei YH. Mitochondrial biogenesis and mitochondrial DNA maintenance of mammalian cells under oxidative stress. Int J Biochem Cell Biol. 2005;37(4):822-34

5. Chen CM, Wu YR, Cheng ML, Liu JL, Lee YM, Lee PW, Soong BW, Chiu DT. Increased oxidative damage and mitochondrial abnormalities in the peripheral blood of Huntington's disease patients. Biochem Biophys Res Commun. 2007;359(2):335-40.

6. Lee $\mathrm{HC}$, Yin $\mathrm{PH}, \mathrm{Lu} \mathrm{CY}$, Chi CW, Wei YH. Increase of mitochondria and mitochondrial DNA in response to oxidative stress in human cells. Biochem J. 2000;348(Pt 2):425-32.

7. Liu CS, Tsai CS, Kuo CL, Chen HW, Lii CK, Ma YS, Wei YH. Oxidative stressrelated alteration of the copy number of mitochondrial DNA in human leukocytes. Free Radic Res. 2003:37(12):1307-17.

8. Lattuada D, Colleoni F, Martinelli A, Garretto A, Magni R, Radaelli T, Cetin I. Higher mitochondrial DNA content in human IUGR placenta. Placenta. 2008; 29(12):1029-33.

9. Mando C, De Palma C, Stampalija T, Anelli GM, Figus M, Novielli C, Parisi F, Clementi E, Ferrazzi E, Cetin I. Placental mitochondrial content and function in intrauterine growth restriction and preeclampsia. Am J Physiol Endocrinol Metab. 2014;306(4):E404-13.

10. von Kleist-Retzow JC, Cormier-Daire V, Viot G, Goldenberg A, Mardach B, Amiel J, Saada P, Dumez Y, Brunelle F, Saudubray JM, et al. Antenatal manifestations of mitochondrial respiratory chain deficiency. J Pediatr. 2003;143(2):208-12.

11. Couck I, Lewi L. The placenta in twin-to-twin transfusion syndrome and twin Anemia polycythemia sequence. Twin Res Hum Genet. 2016;19(3):184-90.

12. Quintero RA, Morales WJ, Allen MH, Bornick PW, Johnson PK, Kruger M. Staging of twin-twin transfusion syndrome. J Perinatol. 1999;19(8 Pt 1):550-5.

13. Chang YL, Chao AS, Chang SD, Hsieh PC, Wang CN. Short-term outcomes of fetoscopic laser surgery for severe twin-twin transfusion syndrome from Taiwan single center experience: demonstration of learning curve effect on the fetal outcomes. Taiwan J Obstet Gynecol. 2012;51(3):350-3.

14. Grubbs BH, Benirschke K, Korst LM, Llanes A, Yedigarova L, Chmait RH. Role of low placental share in twin-twin transfusion syndrome complicated by intrauterine growth restriction. Placenta. 2011;32(8):616-8.

15. Chang YL, Chang SD, Chao AS, Hsieh PC, Wang CN, Wang TH. Clinical outcome and placental territory ratio of monochorionic twin pregnancies and selective intrauterine growth restriction with different types of umbilical artery Doppler. Prenat Diagn. 2009;29(3):253-6.

16. Chang YL, Wang CN, Wei PC, Peng HH, Chao AS, Chang SD, Cheng PJ, Wang TH. Mitochondrial activation in the growth-restricted fetus of monochorionic twins. Fertil Steril. 2013;100(1):241-246 e241-242.

17. Ananth CV, Vintzileos AM, Shen-Schwarz S, Smulian JC, Lai YL. Standards of birth weight in twin gestations stratified by placental chorionicity. Obstet Gynecol. 1998;91(6):917-24.

18. Quintero RA, Martinez JM, Lopez J, Bermudez C, Becerra C, Morales W, Arroyo J. Individual placental territories after selective laser photocoagulation of communicating vessels in twin-twin transfusion syndrome. Am J Obstet Gynecol. 2005;192(4):1112-8.
19. Schmittgen TD, Livak KJ. Analyzing real-time PCR data by the comparative C(T) method. Nat Protoc. 2008:3(6):1101-8.

20. Roberts D, Neilson JP, Kilby MD, Gates S. Interventions for the treatment of twin-twin transfusion syndrome. Cochrane Database Syst Rev. 2014;1: CD002073.

21. Van Winden KR, Quintero RA, Kontopoulos EV, Korst LM, Llanes A, Chmait $\mathrm{RH}$ : Perinatal survival in cases of twin-twin transfusion syndrome complicated by selective intrauterine growth restriction. J Matern Fetal Neonatal Med. 2015, 28(13):1549-1553.

22. Quintero RA, Ishii K, Chmait RH, Bornick PW, Allen MH, Kontopoulos EV. Sequential selective laser photocoagulation of communicating vessels in twintwin transfusion syndrome. J Matern Fetal Neonatal Med. 2007;20(10):763-8.

23. Chang YL, Chang SD, Chao AS, Hsieh PC, Wang CN, Tseng LH. The individual fetal weight/estimated placental weight ratios in monochorionic twins with selective intrauterine growth restriction. Prenat Diagn. 2008:28(3):217-21.

24. Van Winden KR, Quintero RA, Kontopoulos EV, Korst LM, Llanes A, Chmait RH: Perinatal survival in cases of twin-twin transfusion syndrome complicated by selective intrauterine growth restriction. J Matern Fetal Neonatal Med. 2014:1-17.

\section{Submit your next manuscript to BioMed Central and we will help you at every step:}

- We accept pre-submission inquiries

- Our selector tool helps you to find the most relevant journal

- We provide round the clock customer support

- Convenient online submission

- Thorough peer review

- Inclusion in PubMed and all major indexing services

- Maximum visibility for your research

Submit your manuscript at www.biomedcentral.com/submit
Biomed Central 